\title{
The genetic architecture of temperature adaptation is shaped by population ancestry and not by selection regime
}

\author{
Kathrin A. Otte ${ }^{1,2 \dagger} \mathbb{B}$, Viola Nolte ${ }^{1 \dagger}$, François Mallard ${ }^{1,3}$ and Christian Schlötterer ${ }^{1 *}$
}

\author{
* Correspondence: christian. \\ schloetterer@vetmeduni.ac.at \\ ${ }^{\dagger}$ Kathrin A. Otte and Viola Nolte \\ contributed equally to this work. \\ ${ }^{1}$ Institut für Populationsgenetik, \\ Vetmeduni Vienna, Vienna, Austria \\ Full list of author information is \\ available at the end of the article
}

\begin{abstract}
Background: Understanding the genetic architecture of temperature adaptation is key for characterizing and predicting the effect of climate change on natural populations. One particularly promising approach is Evolve and Resequence, which combines advantages of experimental evolution such as time series, replicate populations, and controlled environmental conditions, with whole genome sequencing. Recent analysis of replicate populations from two different Drosophila simulans founder populations, which were adapting to the same novel hot environment, uncovered very different architectures-either many selection targets with large heterogeneity among replicates or fewer selection targets with a consistent response among replicates.
\end{abstract}

Results: Here, we expose the founder population from Portugal to a cold temperature regime. Although almost no selection targets are shared between the hot and cold selection regime, the adaptive architecture was similar. We identify a moderate number of targets under strong selection (19 selection targets, mean selection coefficient $=$ 0.072 ) and parallel responses in the cold evolved replicates. This similarity across different environments indicates that the adaptive architecture depends more on the ancestry of the founder population than the specific selection regime.

Conclusions: These observations will have broad implications for the correct interpretation of the genomic responses to a changing climate in natural populations.

Keywords: Experimental evolution, Temperature adaptation, Adaptive architecture

\section{Introduction}

Adaptation of natural populations to environmental change may either occur from standing genetic variation or by the acquisition of new mutations. The relative importance of these two paths crucially depends on the underlying adaptive architecture [1] of the focal trait. The adaptive architecture differs from the genetic architecture, which is inferred by QTL mapping and GWAS [2, 3], by accounting for pleiotropic constraints as well as for the large body of deleterious mutations $[4,5]$.

(c) The Author(s). 2021 Open Access This article is licensed under a Creative Commons Attribution 4.0 International License, which permits use, sharing, adaptation, distribution and reproduction in any medium or format, as long as you give appropriate credit to the original author(s) and the source, provide a link to the Creative Commons licence, and indicate if changes were made. The images or other third party material in this article are included in the article's Creative Commons licence, unless indicated otherwise in a credit line to the material. If material is not included in the article's Creative Commons licence and your intended use is not permitted by statutory regulation or exceeds the permitted use, you will need to obtain permission directly from the copyright holder. To view a copy of this licence, visit http://creativecommons.org/licenses/by/4.0/. The Creative Commons Public Domain Dedication waiver (http://creativecommons.org/publicdomain/zero/1.0/) applies to the data made available in this article, unless otherwise stated in a credit line to the data. 
Selection targets (Table 1) are identified with genomic selection scans, which apply statistical tests to detect selection signatures from population polymorphism data [7, 8]. Some selection scans assume that selection targets are shared among populations exposed to the same environment, because such parallel signatures provide additional statistical support [9-11]. Many selection targets were successfully identified with these strategies and have contributed to our understanding of the molecular basis of adaptation processes (e.g. [11-15]). It is, nevertheless, not apparent to what extent these results can be generalized, because most adaptive traits have a polygenic basis $[16,17]$ and either only small allele frequency changes [17] or non-parallel responses are expected [1].

The key concept of polygenic adaptation is that multiple loci are contributing to the phenotype, and rather than focusing on particular loci, the collective effect of all loci needs to be considered to estimate the phenotypic value of a given trait. The infinitesimal model [18-20] is the most extreme case of polygenic adaptation and is frequently approximated by very many contributing loci (Table 1 ), each of very small effect. When many loci are contributing to a phenotype under stabilizing selection, any selection regime changing the trait optimum will result only in very small allele frequency shifts $[17,19]$ - almost impossible to detect with classic population genetic tests [21-23].

Even when these conditions are relaxed and a distribution of effect sizes with some large effects is considered, no pronounced allele frequency changes are expected when the populations are large and in mutation selection equilibrium: targets with large effects are segregating at low frequencies only and do not contribute much to the phenotypic variance of the population upon which selection is operating [24, 25]. Theory predicts that as the pool of loci contributing to the selected phenotype becomes smaller (i.e., a decreased mutational target), larger allele frequency changes are expected that will be detected in population genetic analyses [26]. Therefore, traits with an intermediate number of contributing loci are particularly interesting, because the response of these loci can be sufficiently strong to be detected in experiments while, at the same time, being informative about polygenic adaptation: more loci are segregating in the population than required to reach a new trait optimum (i.e., genetic redundancy).

The consequence of this genetic redundancy is that the contribution to the phenotype can be highly heterogeneous for individual loci in differentiated populations if they vary in frequency. This expectation nicely conforms with empirical data, mostly from QTL studies, which find heterogeneous sets of contributing loci among different populations

Table 1 Definition of frequently used terms

\begin{tabular}{ll}
\hline Term & Definition \\
\hline Contributing loci & $\begin{array}{l}\text { Loci, which are affecting the trait irrespective of whether they are responding to } \\
\text { selection or not (hence we use contributing loci only in the context of genetic } \\
\text { architecture, but not adaptive architecture) }\end{array}$ \\
Selection targets & $\begin{array}{l}\text { A generic term for loci or SNPs, which are responding to selection (i.e. a subset of the } \\
\text { contributing loci) }\end{array}$ \\
Genomic region & $\begin{array}{l}\text { Describes a physical area of the genome, but does not consider possible associations } \\
\text { between alleles located there }\end{array}$ \\
(Selected) haplotype & $\begin{array}{l}\text { Genomic region with SNPs in linkage disequilibrium that undergo correlated allele } \\
\text { block } \\
\text { frequency changes during the experiment; it is the smallest unit under selection that can } \\
\text { be experimentally resolved-it may contain one or multiple selected SNPs. Haplotype } \\
\text { blocks are identified and characterized by SNPs with a correlated allele frequency change } \\
\text { after multiple generations of experimental evolution [6]. }\end{array}$ \\
\hline
\end{tabular}


[27-36]. In the case of adaptation to a new trait optimum, genetically differentiated populations will adapt by frequency changes of different sets of loci. Hence, for polygenic adaptation, the identity of individual selection targets is not very important to describe the adaptive architecture, rather information about the number of loci, effect sizes, and frequencies is needed to understand the selective response.

Selection signatures not shared among natural populations are difficult to interpret, as the distinction between population-specific selection targets and false positive/negative signals can be challenging given the high impact of a largely obscure demography on selection signatures [37-42]. Experimental evolution, in contrast, provides the advantage of replicate populations, which evolve from the same founder population under controlled experimental conditions [43]. The potential of experimental evolution to study the genomic signatures of polygenic adaptation has, however, not yet been fully exploited since most studies apply truncating selection. Thus, the contributing loci experience continued selection pressure throughout the entire experiment, causing a parallel selection response in the replicate populations towards an extreme phenotype. Laboratory natural selection is a specific experimental evolution design, where the evolving populations are exposed to a new environment [44]. In contrast to truncating selection, populations are expected to reach a new phenotypic optimum, thus selection depends on the distance to the trait optimum. In combination with whole genome sequencing, it provides an interesting approach to study the adaptive architecture experimentally.

Two previous experimental evolution studies conducted in the same novel hot laboratory environment showed parallel fitness increase among replicates (see Additional file 1: Figure S1), but the genomic analysis revealed very different adaptive architectures [45, 46]. In the Portugal experiment, five strongly selected targets were identified and this selection signal was highly parallel across replicates. For the Florida experiment, 99 selection targets were identified and considerable heterogeneity was observed between the replicates. Several explanations for this different adaptive architecture are conceivable, including different ancestral trait optima of the two founder populations [47], or more large effect alleles segregating at higher frequencies in the Portugal founder population.

Here, we exposed replicate populations of the Portugal founders to a cold temperature regime to shed more light on the different selection responses. Interestingly, we found very little overlap between the genomic position of the selection targets in the hot and cold temperature regimes. Most large effect selection targets in the hot environment did not respond in the cold, suggesting that hot and cold temperature adaptation may be different traits, rather than a simple shift in optimum of the trait "temperature adaptation." Nevertheless, adaptation to both, hot and cold, temperature regimes had a very similar adaptive architecture-with a comparable number of selection targets and effect sizes. We conclude that the adaptive architecture differs between populations and may be trait independent. We discuss to what extent this phenomenon can be explained by the infinitesimal model.

\section{Results}

We studied the genetic architecture of cold adaptation in Drosophila simulans by combining experimental evolution with whole genome re-sequencing (Evolve and Resequence, E\&R). Five replicate populations originating from the Portuguese founder population described by Mallard F, Nolte V, Tobler R, Kapun M, and Schlötterer C [46] 
evolved for more than 50 generations (about 4 years) in a cold temperature regime with daily fluctuations between 10 and $20^{\circ} \mathrm{C}$. Genome-wide allele frequencies were determined in 10 generation intervals by sequencing pools of individuals (Pool-Seq [48]). Contrasting generation 0 with 51 we identified 6527 SNPs, which changed in frequency more than expected by genetic drift either across all five replicates (adapted $\mathrm{CMH}$ test [49], $6510 \mathrm{SNPs}$ ) or at least in one replicate (adapted $\chi^{2}$ test [49], additional $17 \mathrm{SNPs}$ ). The X chromosome harbored only 142 SNPs. Such a low number of candidate SNPs on this chromosome was not seen in other Drosophila E\&R studies that observed similar numbers of candidate SNPs on the X chromosome and autosomes [45, 50-53].

The pronounced peak structure in the Manhattan plot (Fig. 1A) indicates that many candidate SNPs are not independent due to linkage [54,55]. We accounted for this and employed a correlation-based haplotype reconstruction approach to identify independently selected haplotype blocks based on their distinct trajectories [54, 56] and treated each of these haplotype blocks as a single selection target [45].

\section{Selected haplotype blocks in the cold-evolved $D$. simulans population}

Because the small number of candidate SNPs precluded haplotype block reconstruction on the $\mathrm{X}$ chromosome, all 15 haplotype blocks were identified on the major autosomes (Fig. 1B, C). The size of the haplotype blocks ranged from $5 \mathrm{~kb}$ to $6 \mathrm{Mb}$. Three of the 15 blocks were located on chromosome 2 while all others fell on chromosome 3.

The 15 selected haplotype blocks were identified by clustering SNPs with similar allele frequency trajectories in the five replicates and six time points. A conservative assumption is that each of the selected haplotype blocks contains one selection target. Nevertheless, multiple selection targets can recombine during the experiment onto a single haplotype block, which outcompetes the individual parental haplotype blocks [56]. Such multiple-target haplotypes will dominate the later phases of the experiment and are considered as a single selected haplotype. To identify such cases, we repeated the haplotype block reconstruction with fewer generations-four time points up to generation 31 -and found that two reconstructed haplotype blocks could be further broken up: block 4 and 10 were split into three different blocks each (4a, 4b, 4c and 10a, 10b, 10c, see Fig. 1C). This observation confirms that the number of inferred independent haplotype blocks is a conservative estimate of the number of selection targets, and we used the sub-blocks for subsequent analyses whenever applicable, analyzing a total of 19 selection targets.

Selected haplotype blocks are characterized by a set of marker SNPs which show correlated allele frequency trajectories across replicates. Nevertheless, the correlation threshold is not very stringent to account for sequence diversity among the haplotypes carrying the selection target [56]. Hence, not all of the haplotype block marker SNPs describe the frequency trajectory of the (unidentified) selection target equally well. Reasoning that SNPs with the most pronounced allele frequency change are the best representatives of the selection target, we used the $10 \%$ most significant marker SNPs of each haplotype block and refer to them as the selection target. The frequency of each selection target at every time point is determined as the median frequency of these $10 \%$ of most significant SNPs. We used only replicates with a selection coefficient large enough to be significantly different from neutrality ( $p$-value $<0.05$ ), therefore we excluded replicates in which a given selection target did not increase in frequency. The 


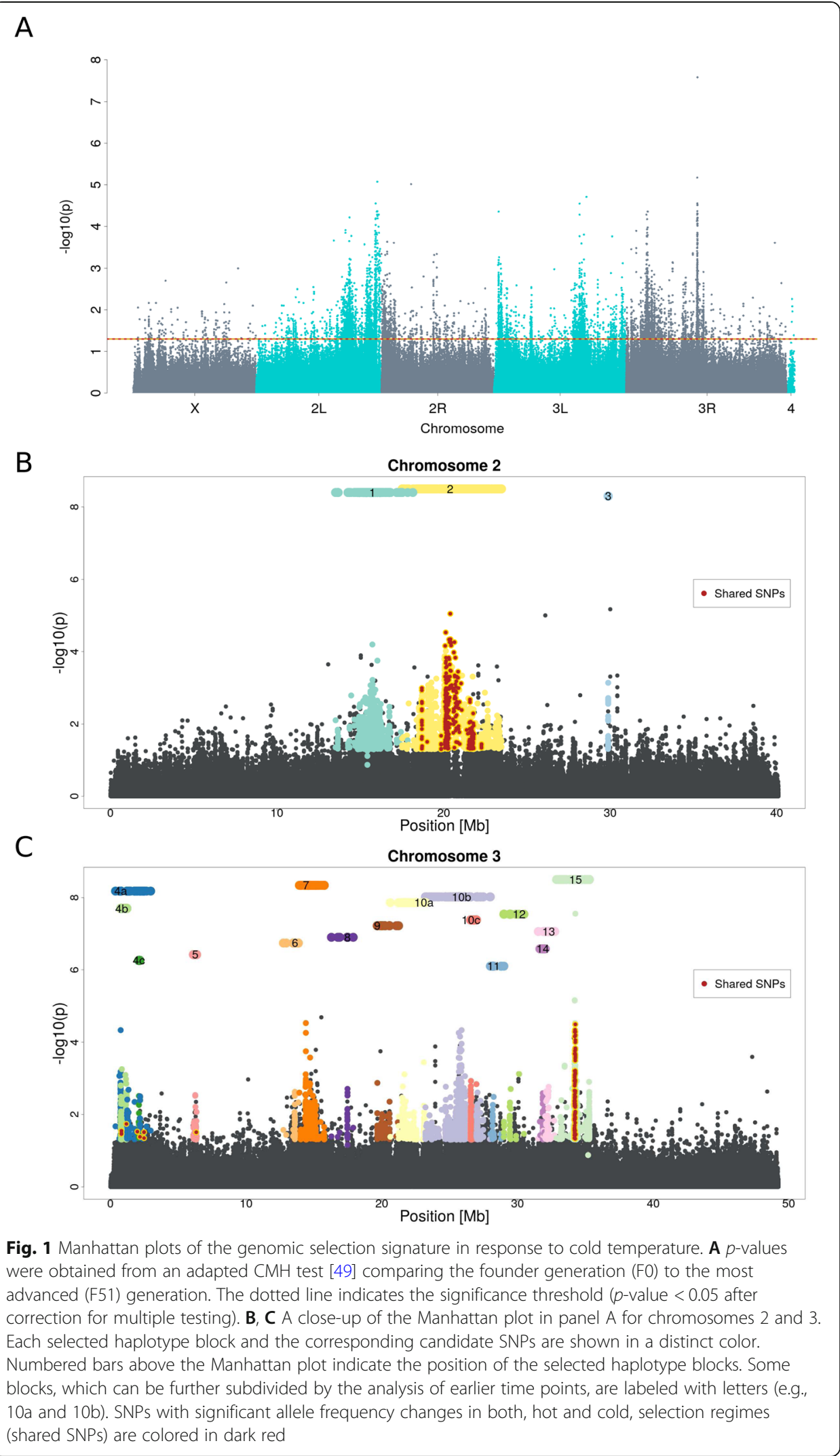


starting frequencies were highly variable among the 19 selection targets. We detected selection targets with starting frequency as low as 0.06 , but also as high as 0.4 (Fig. 2A). The selection coefficients were rather high and ranged from 0.04 to 0.11 (Fig. 2A).

Overall, we noticed a striking relationship between starting frequency and selection coefficients. Selection targets starting with lower frequencies had higher selection coefficients than those with higher starting frequencies. This relationship was significant when analyzing the full set of 19 blocks including the broken-up haplotype blocks (i.e., replacing blocks 4 and 10 by sub-blocks $4 \mathrm{a}, 4 \mathrm{~b}, 4 \mathrm{c}$ and 10a, 10b, 10c) and was not significantly influenced by block size (linear regression; factor: starting allele frequency $p=0.004$ and factor: block size $p=0.130$ ). The inverse relationship between starting allele frequency and selection coefficient was robust with respect to the definition of a selection target (see Additional file 1: Figure S2).

While a negative correlation between frequency and effect size is expected by theory and has been previously reported for GWAS [5, 57-59] and E\&R [45] studies, it is important to note that a Beavis-like effect $[60,61]$ may also contribute to this observation: alleles with low starting frequencies require stronger selection to result in detectable allele frequency changes than alleles starting from intermediate frequencies.

With a median size of $1.5 \mathrm{Mb}$, the selected haplotype blocks were rather large. The median number of genes per selected haplotype block is 20 but it can reach up to 154 (see Additional file 1: Figure S3) in the largest reconstructed block (block 2, see Fig. 1B). The smallest block contained only a single candidate gene (block number $3,4.8$ $\mathrm{kb})$. All 23 marker SNPs were located within one intronic region of the gene M-spondin (mspo, FBgn0020269), an extra-cellular matrix protein of Drosophila, putatively involved
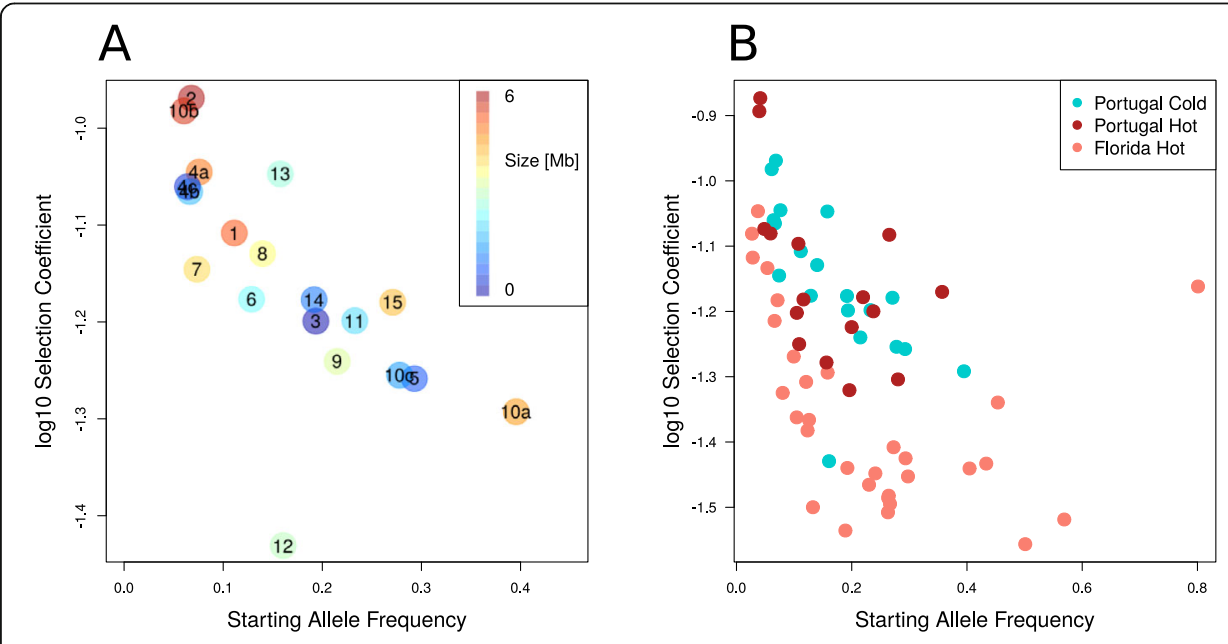

Fig. 2 Inverse relationship between starting allele frequency and selection strength. For all experiments, the selection targets were identified using the same protocol and are based on the top 10\% SNPs in reconstructed haplotypes. A The relationship is shown for the Portugal cold experiment. The color code reflects the size of the selected haplotype block, and starting allele frequencies and selection coefficient of the selection targets are plotted on $\mathrm{x}$ - and $\mathrm{y}$-axis. The numbers relate to the selected haplotype blocks from Fig. $1 \mathrm{~B}$ and $\mathrm{C}$ with numbers indicating blocks detected in the analysis using all time points. Sub-blocks resulting from an analysis using earlier time points are labeled with the letters $\mathrm{a}$, b, or c. B A qualitatively similar relationship between starting allele frequency of each haplotype block and selection strength is found in the cold-evolved Portugal (blue), hot-evolved Portugal (red), and hot-evolved Florida (pink) population. Nevertheless, the distribution in the Florida experiment was shifted towards lower selection coefficients while the two temperature regimes in the Portugal population were highly similar. We conclude that the adaptive architecture is population-specific but does not depend on the temperature regime 
in muscle development [62]. The role of this gene in temperature adaption is not apparent and further studies are required for a better understanding of this selection signature.

\section{Temperature-specific adaptation}

Replicates from the same Portuguese founder population were also exposed to a hot selection regime fluctuating between $18^{\circ} \mathrm{C}$ and $28^{\circ} \mathrm{C}$ [46]. Both temperature regimes have the same daily temperature amplitude of $10^{\circ} \mathrm{C}$ (cold $10 / 20^{\circ} \mathrm{C}$ and hot $18 / 28^{\circ} \mathrm{C}$ ), but mean temperatures differ $\left(15^{\circ} \mathrm{C}\right.$ in the cold and $23^{\circ} \mathrm{C}$ in the hot regime). The temperatures were chosen such that one of the temperatures is stressful, whereas the other temperature is benign $[63,64]$. Contrasting the founder population with hotevolved generation 59, Mallard F, Nolte V, Tobler R, Kapun M, and Schlötterer C [46] identified few (five) very pronounced selection peaks, some of them related to metabolic alterations in the hot-evolved populations.

For an unbiased comparison of the two experiments, we added time series data for the hot-evolved populations (F0, F15, F37, F59) and applied the same haplotype reconstruction pipeline as described above. Similar to the cold-evolved population, the $\mathrm{X}$ chromosome had too few outlier SNPs (114 SNPs) for haplotype reconstruction. 16 selected haplotype blocks were identified on the two major autosomes (Additional file 1: Figure S4) and their selection coefficients ranged from 0.05 to 0.13 (Fig. 2B). It is remarkable that not only the number of inferred selection targets, but also the distribution of selection coefficients is highly similar for the two temperature regimes (hotevolved $=16$ blocks, cold-evolved $=19$ blocks).

Only two haplotype blocks shared more SNPs than expected by chance between the two temperature regimes. Furthermore, the shared region was in both cases only a small part of the total haplotype block (Fig. 1B, C; blocks 2 and 15). A prominent similarity between the selection regimes could be identified in block 15, where the majority of overlapping SNPs were located in the gene Ace (FBgn000024). Nevertheless, the selection pattern for Ace differs between both temperature regimes [65]. The shared SNPs of block 2 were located in several genes, and therefore no clear candidate for common adaptation could be identified in this region.

We further scrutinized the haplotype blocks that were not shared between the selection regimes and had a starting frequency higher than 0.15 to rule out that a selection signature in opposite direction-as expected for a polygenic trait selected in contrasting environments-was missed. The allele frequency change of all candidate SNPs in a haplotype block was always higher in the focal temperature regime. Importantly, in both, hot and cold, selection regimes we very rarely observed a frequency change in the opposite direction (Additional file 1: Figure S5). We conclude, therefore, that we have no support for alleles being selected in opposite direction in hot and cold temperatures. Rather, most alleles show a temperature-specific response.

\section{Population-specific adaptation}

Independent of the temperature regime the evolved populations derived from the Portugal founder population revealed only a moderate number of selection targets. This contrasts a recent experiment using the same hot temperature regime but a founder population from Florida [45]. For a consistent comparison to the Portugal population, 
we repeated the analysis of the Florida population using our haplotype reconstruction pipeline but focused only on the two major autosomes. 31 selected haplotype blocks were identified on chromosome 2 and 3 (Additional file 1: Figure S6), which are considerably fewer selection targets than the 88 reported by Barghi N, Tobler R, Nolte V, Jaksic AM, Mallard F, Otte KA, Dolezal M, Taus T, Kofler R, and Schlötterer C [45] for these two chromosomes. This difference reflects an alternative strategy to identify candidate SNP sets for the haplotype reconstruction rather than the clustering method [56]. Following the same protocol as for the Portugal experiments, 9197 outlier SNPs were identified, which is more conservative than the 52,199 outlier SNPs used by Barghi N, Tobler R, Nolte V, Jaksic AM, Mallard F, Otte KA, Dolezal M, Taus T, Kofler R, and Schlötterer C [45] for haplotype reconstruction. The Florida population harbored about twice as many selected haplotype blocks as the hot-evolved Portugal population. For all experiments, we identified the same relationship between starting frequency and selection coefficients, but the distribution for Portugal was shifted towards higher selection coefficients (Fig. 2B). This result is robust with respect to the definition of selection target (see Additional file 1: Figure S7). This difference in selection coefficients remains significant when we account for allele frequencies in the founder populations (contrasts between estimated marginal means Portugal cold - Florida hot $p<0.0001$ and Portugal hot - Florida hot $p<$ 0.0001). No significant difference was observed between the two temperature regimes of the Portugal population (Portugal cold - Portugal hot $p=0.98$ ).

The Florida experiment was based on twice the number of replicates as the Portugal experiment. To rule out that the number of replicates affects the inferred adaptive architecture, we repeated the analysis with 100 sets of five randomly sampled Florida replicates. In all 100 random subsets, the selection coefficient was not different from the full data set (Wilcoxon rank-sum test $p>0.05$ adjusted for multiple testing using the Benjamini-Hochberg method), suggesting that the difference between the Portugal and Florida data sets cannot be explained by a different number of replicates.

We quantified the degree of parallelism between the experiments using the Jaccard index, creating a binary presence/absence matrix of selected haplotype blocks with $s$ significantly different from zero among replicates. High values indicate parallel genetic responses whereas low values reflect heterogeneous, non-parallel genetic responses between replicates. Jaccard indices were high for the two Portugal experiments (median similarity between replicates $80 \%$ and $82 \%$, respectively). For the Florida experiment, the Jaccard index was significantly lower (median similarity between replicates $70 \%$ for the full data set; Wilcoxon rank-sum test $p$-value $<0.001$ against each Portugal data set), indicating less parallel genetic responses and therefore increased realized genetic redundancy. This pattern was robust with respect to the method used to define a selection target in a given replicate (Fig. 3 and Additional file 1: Figure S8).

The comparison of selected haplotype blocks between evolved populations derived from different founder populations is difficult because the selected haplotype blocks are reconstructed only for significant SNPs and the full sequence of the selected haplotype block remains unknown. Even if the selected haplotype block is shared in both populations, the remaining, non-selected, haplotypes differ between the populations. Hence, as 


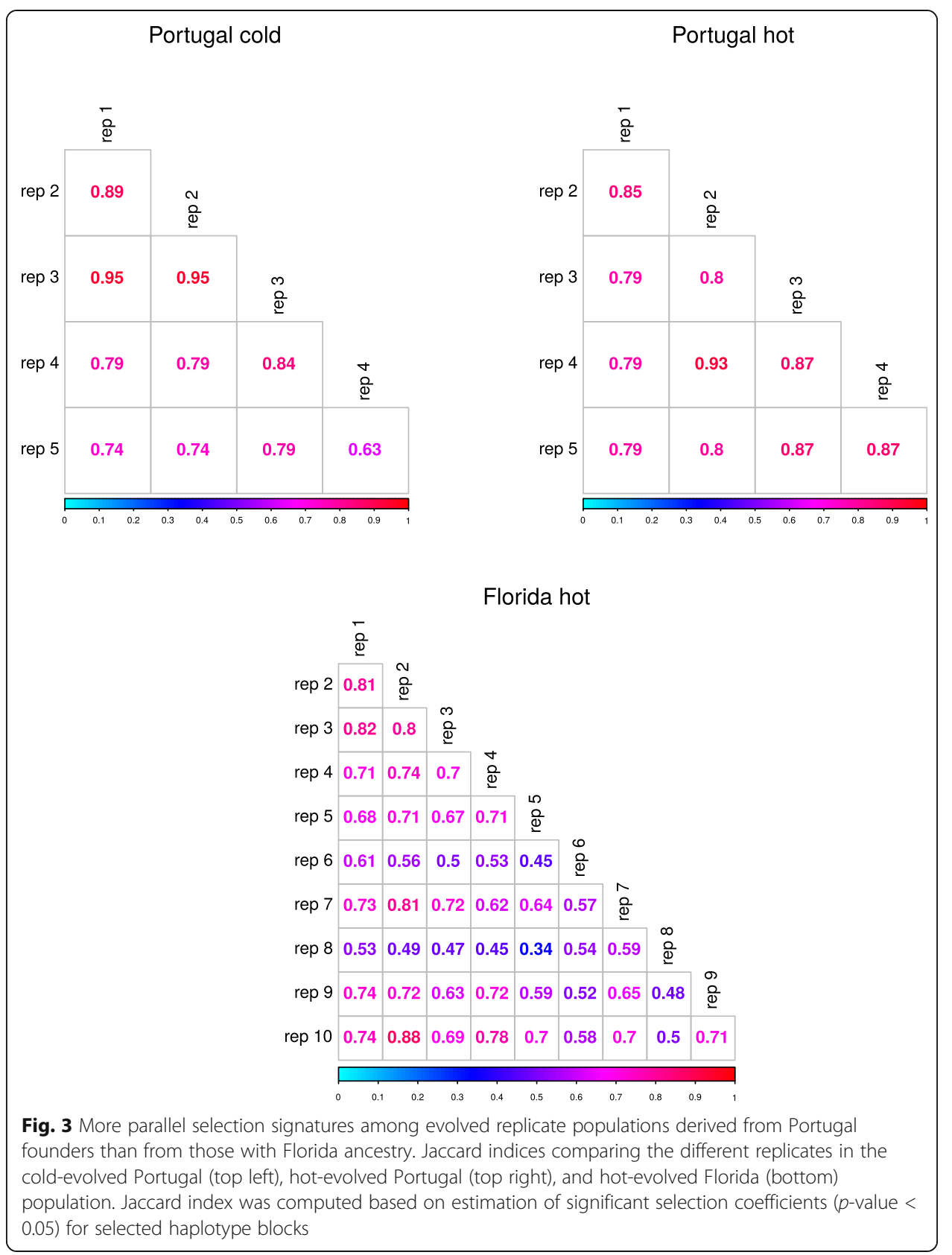

the frequency change at a given SNP on the selected haplotype depends on the frequency of this SNP in the non-selected haplotypes, different candidate SNPs could be identified in the two populations- even if the same haplotype block is selected. Thus, the same selected haplotype block may have different marker SNPs in two different populations - suggesting that different haplotype blocks are selected. Furthermore, the low linkage disequilibrium in natural Drosophila populations [66, 67] implies that very few haplotypes are expected to be shared between samples from different populations. For these reasons, we did not attempt to test whether the same haplotype blocks are selected in Portugal and Florida. 


\section{Discussion}

D. simulans populations of different origins (Portugal and Florida) had very distinct adaptive architectures in the same hot temperature regime [45, 46]: Portugal had fewer selection targets, which were strongly selected in a highly parallel manner. Florida harbored more selection targets with more heterogeneity among replicates and lower selection coefficients compared to Portugal. In this report, we studied replicate populations derived from the Portugal founder population, which adapted to a cold temperature regime to understand why such different adaptive architectures were inferred in these two populations. Below, we discuss several possible explanations for the differences in adaptive architecture.

\section{Different trait optima in the ancestral populations}

Both founder populations were collected on different locations with their own specific temperature profile and in different phases of the seasonal cycle (Portugal in July 2008, Florida in November 2010). Assuming that temperature adaptation is a single high-level trait, the ancestral trait optimum may differ on the phenotypic axis. This implies that a population, which is less adapted to hot environments should be better adapted to cold environments. Less well-adapted populations will experience stronger and more parallel selection responses across replicates $[1,68]$, consistent with the pattern observed in the hot Portugal population. Different trait optima of the founder populations were further supported by the observation that the Portugal founder population is less fecund than the Florida founder population when assayed in the novel hot environment (Barghi et al., unpublished results). This implies that the mean phenotype of the Portugal population is more distant from the new trait optimum in the hot laboratory environment than the Florida population.

The analysis of the cold-evolved replicates casts some doubts on this simple interpretation. We assumed that the hot and cold experiments shifted the trait optimum into opposite directions relative to the (unknown) trait optimum of the ancestral Portugal population. Hence, contributing loci segregating at sufficiently high frequency in the ancestral population should be selected in opposite direction in the two temperature regimes. Nevertheless, the results did not fit our expectations-most selected haplotype blocks were not shared between the two temperature regimes. While selected haplotype blocks starting from low frequencies may not be identified as selection targets in the opposite temperature regime, also haplotype blocks with higher allele frequencies in the founder population were not selected in opposite direction.

Hence, we conclude that temperature adaptation may not be a single high-level phenotype. Rather, several sub-phenotypes on a lower level, which are not all shared for the different temperature regimes, are contributing to adaptation. This conclusion is further supported by different genomic signatures of hot and cold stressors in E\&R [69] and QTL mapping [70] studies.

\section{Differences in adaptive variation}

Autosomal polymorphism levels differ between the two founder populations with Florida being more variable than Portugal $\left(\pi_{\text {Florida }}=0.0076\right.$ and $\pi_{\text {Portugal }}=0.0062$, Wilcoxon rank-sum test on non-overlapping $10 \mathrm{~kb}$ windows, $p$-value $<0.001$ ). Assuming that neutral variability is a good approximation of adaptive variation, which is not 
always the case [71], Portugal is expected to harbor less adaptive variation than Florida. This implies that Florida reaches the trait optimum faster than Portugal [47, 72], but in absence of phenotypic time series data, we cannot assess this hypothesis. A particularly interesting hypothesis related to the different polymorphism levels is that Portugal harbors so little adaptive variation that it does not have much genetic redundancy. This would imply that no (or only limited) excess of adaptive genetic variation is segregating in the Portugal founder population that can be used to reach the trait optimum.

The Florida founder population, in contrast, harbors a considerable excess. Such differences in the number of contributing loci can generate quite different patterns of parallel selection responses [47], matching the Portugal and Florida experiments. Nevertheless, it is not apparent that the moderate differences in genome-wide polymorphism levels are sufficiently large to explain this pattern.

\section{Linkage disequilibrium}

The above discussion about the heterogeneity of the inferred genetic architectures between populations and selection regimes rests on the central assumption that the major contributing loci were identified and could be distinguished with a recently developed haplotype reconstruction approach [56]. In other words, it is assumed that only a moderate number of distinct loci contribute to adaptation.

Alternatively, the observed selection response may be explained by many loci of small effect - an idea that matches in its extreme form the infinitesimal model [18]. Empirical support for a highly polygenic architecture of many traits comes from the strong correlation between chromosome length and the fraction of heritability explained [73-75]. If multiple small effect loci cluster together this may result in a signature that will be interpreted as a single selection target [76]. Short genomic regions with a local clustering of contributing loci can even introgress and leave the strong selection signature of a local allele frequency change [77]. Empirical support for the clustering of contributing loci comes from the molecular dissection of candidate loci identified in QTL mapping studies. Single QTL loci can be broken into multiple SNPs contributing to the corresponding trait [78-83].

The situation in polymorphic founder populations is significantly more complicated than the simple two genotype case studied by Sachdeva H and Barton NH [77], but we propose that blocks of linked loci can not only generate pronounced selection signatures, but may also explain the differences in adaptive architecture between the Portugal and Florida experiment. Depending on the extent of linkage disequilibrium (LD) the clustering of contributing loci can vary. Hence, populations with different levels of LD may also harbor more or less clustered contributing loci. The influence of haplotype structure can be illustrated by two extreme cases: in the case of complete linkage equilibrium, in any genomic region the haplotypes segregating in the population should have similar fitness effects despite being highly diverse. As a consequence, changes in trait optimum will result only in rather small frequency changes of the haplotypes in this genomic window. This pattern becomes more pronounced with an increasing number of contributing loci.

On the other hand, in the presence of strong linkage disequilibrium, fewer distinct haplotypes are present in a given genomic window. Sampling variation in the ancestral population generates haplotypes with different numbers of contributing loci in a given 


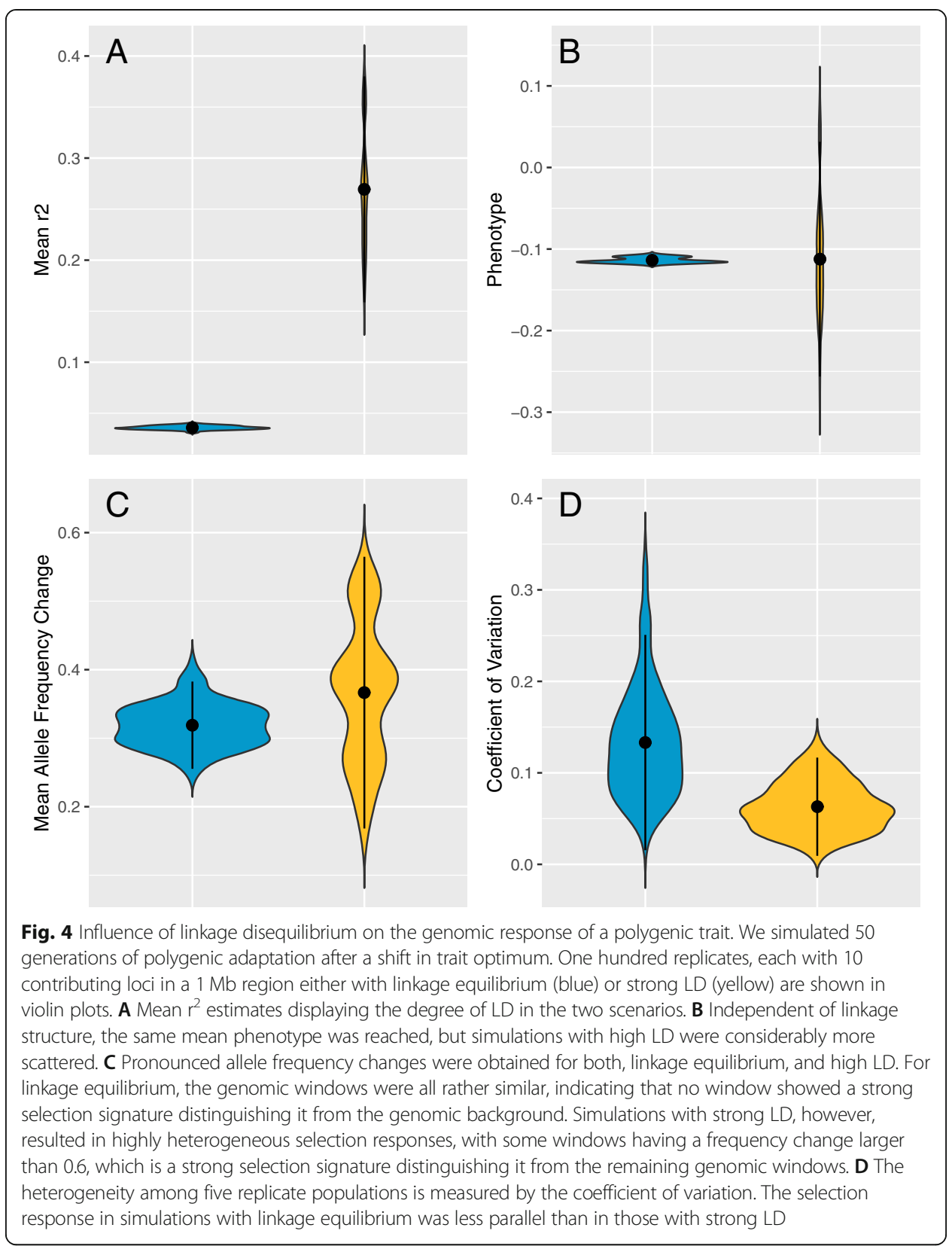

genomic window. The more pronounced the difference in the number of loci among haplotypes in a genomic window is, the stronger will be the differences in fitness effects among them and thus the allele frequency change after a shift in trait optimum. Hence, because the difference in the number of contributing loci among haplotypes differs among genomic windows, linkage disequilibrium generates heterogeneity in selection response along the chromosome.

We illustrated the impact of LD with the help of forward simulations. We simulated adaptation after a shift in trait optimum modeling the associated genotypes, phenotypes, and fitness. The genotypic value of an individual was determined by the additive effect sizes of all contributing loci while the phenotypic value was calculated by incorporating heritability. Fitness was computed based on the phenotypic value and a Gaussian fitness function. 
We simulated 10 contributing loci in a genomic window of $1 \mathrm{Mb}$ in a typical E\&R setting $[84,85]$. For high LD and linkage equilibrium (Fig. 4A) the same number of chromosomes with contributing loci was used. 100 different genomic windows were simulated and simulations with higher LD resulted in a more heterogeneous response to selection. Consistent with a larger phenotypic variance (Fig. 4B) also much more pronounced allele frequency changes were observed for some windows in the presence of linkage disequilibrium (Fig. 4C). Hence, while very homogeneous moderate frequency shifts were observed for contributing loci in linkage equilibrium, some pronounced sweep windows were detected for windows with LD. We also assessed the degree of parallelism in the response between replicates and found a more parallel response for the high LD simulations (Fig. 4D).

Thus, for polygenic traits, the inferred adaptive architecture can be strongly affected by linkage in the ancestral population. The importance of linkage has also been recently emphasized, in particular for genomic regions (Table 1) with suppressed recombination, such as inversions [86]. A previous study on the impact of recombination on the response to a shift in trait optimum with truncating selection observed more heterogeneity among replicates in the case of linkage equilibrium than for complete linkage [87]. We attribute these differences to the small population sizes in [87].

Nevertheless, does this scenario of a highly polygenic architecture with differences in LD apply to the Florida and Portugal experiments? Following the same rationale as $[45,73]$ tested whether longer haplotype blocks were more strongly selected than shorter ones, but no significant correlation was found. Similar results were observed for the cold evolved Portugal populations. We caution, however, that these negative results do not provide strong support for the identification of distinct selection targets. Possible, not mutually exclusive, reasons for the lack of significance even in the presence of a highly polygenic architecture are (1) the contributing loci have different effect sizes, thus small haplotype blocks with large effect contributing loci may be more strongly selected than larger blocks, (2) larger blocks may harbor more contributing loci with effects in opposite direction than smaller ones, and (3) since haplotype blocks are still relatively short (compared to full chromosomes), stochastic sampling and heterogeneity in the density of contributing loci may obscure the correlation between the size of the selected haplotype block and the selection response.

The hypothesis that LD differences can explain the heterogeneity in inferred adaptive architecture is supported by the observation that the two founder populations differed across all chromosomes in their pattern of linkage disequilibrium (Fig. 5). The higher LD in Portugal compared to Florida is fully consistent with the prediction that founder populations with higher LD are more likely to show stronger and more parallel sweep signatures than populations with lower LD. Although this pattern fits our observations, in absence of more information about the degree of polygenicity and the distribution of contributing loci across the chromosomes and their effect sizes, it is not possible to determine whether the observed differences in LD are sufficient to explain our empirical data.

\section{Temperature adaptation may involve multiple, temperature-specific traits}

While we cannot pinpoint the cause for the differences in the inferred adaptive architecture between Portugal and Florida, the analysis of cold-evolved replicates sheds some important light on our understanding of temperature adaptation. 

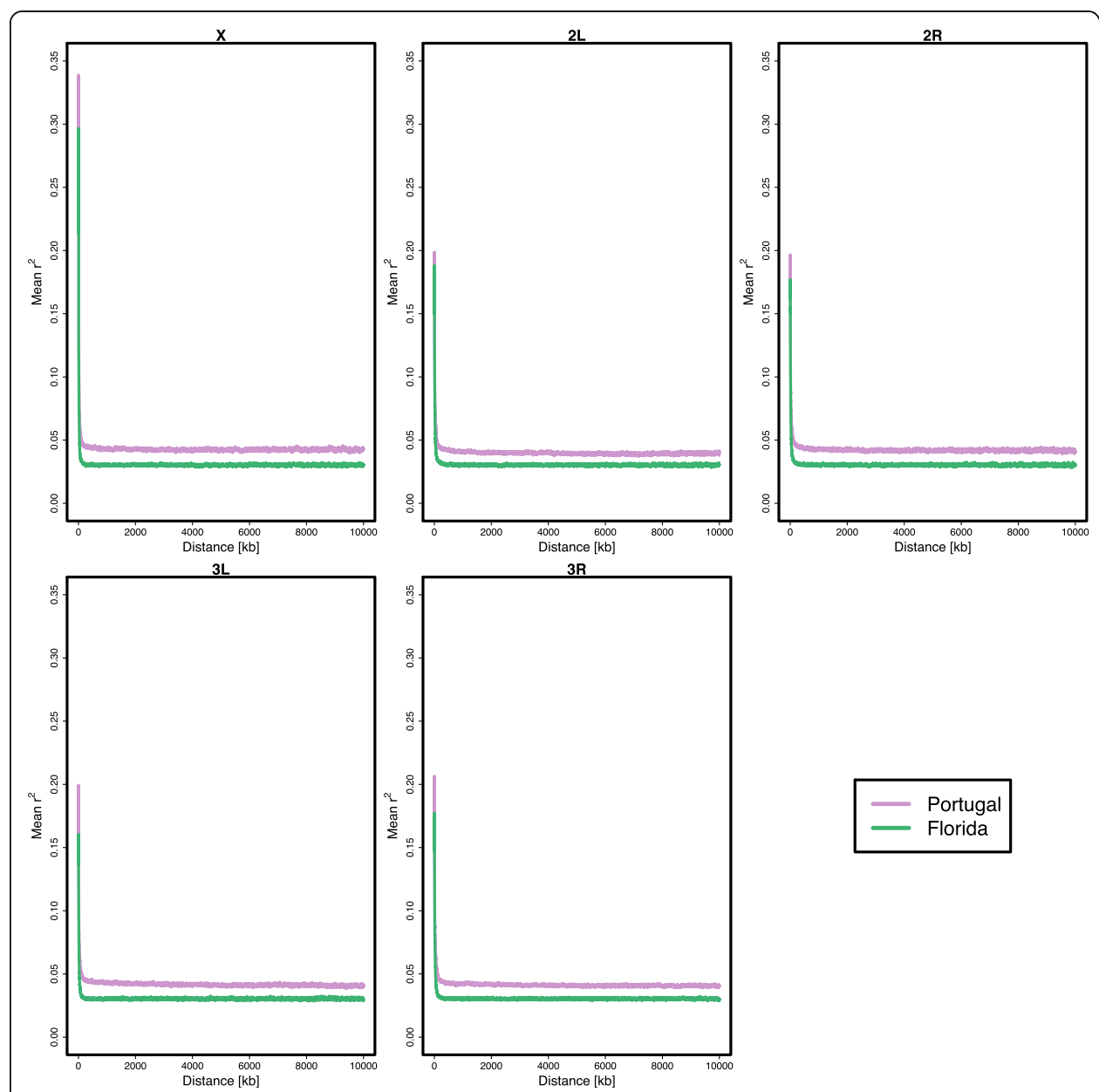

Fig. 5 Linkage disequilibrium in the ancestral Portugal und Florida population as measured by the mean $r^{2}$ of loci with distances up to $10,000 \mathrm{~kb}$ based on 34 individual haplotype sequences

Many studies, in particular theoretical ones, considered high-level phenotypes, such as temperature adaptation, as a single adaptive trait, where shifts in mean temperature are treated as a simple shift in trait optimum (e.g., [88-91]). This implies that all segregating contributing loci affect the optimal phenotype - irrespective of the position of the optimum - i.e. in the case of temperature adaptation irrespective of whether the optimum is in the hot or cold. The limited overlap between the selection targets in hot- and cold-evolved replicates is striking, as it contradicts this assumption. With the exception of the haplotype blocks containing the Ace locus and a haplotype block across the centromere of chromosome 2, which changed in the same direction in both temperature regimes, no shared haplotype blocks were detected. To some extent, the lack of shared haplotype blocks can be attributed to low starting frequencies, which implies that selection in the opposite direction does not result in allele frequency changes sufficiently large to be detected. Nevertheless, even for haplotype blocks starting from intermediate frequency, no selection signature in the opposite direction was noticed.

This implies that different loci are contributing to adaptation in hot and cold environments - irrespective of whether a highly or moderately polygenic architecture is 
assumed. A very similar lack of shared candidates was also noticed in a D. melanogaster experiment, where replicate populations were exposed to the same hot and cold temperature regimes [69]. This experiment was, however, conducted for a much smaller number of generations, and the selection signature was analyzed on the SNP-level, which makes the interpretation of the results particularly challenging given the contribution of large segregating inversions to temperature adaptation in this species [92, 93].

The observation of different selection targets in hot and cold environments is particularly interesting, because seasonal changes were found to be associated with cycling allele frequencies in natural D. melanogaster populations [94], which suggests that the same SNPs are being selected in opposite directions in hot and cold environments. One possible explanation for these differences to our study is that in natural populations only a moderate number of generations separates the two temperature regimes, while in our experiment the temperature regime remained constant across more than 50 generations.

\section{Population-specific adaptive architectures}

Various studies, mainly using QTL mapping and GWAS, have identified different loci contributing to the same trait in diverged populations [27-36, 95]. A recent experimental evolution study using $D$. subobscura populations with different genetic backgrounds also observed very little overlap in the genomic regions responding to a common selection regime [96]. Hence, the different selection targets obtained from Portugal and Florida experiments conducted in the same hot environment are not particularly surprising and emphasize the limited insights about the genetic basis of a polygenic trait from single population studies. Very surprising, however, was the observation that the adaptive architecture (number of contributing loci, effect sizes, and starting frequencies) was different between Portugal and Florida, but strikingly similar between the hot and cold selection regimes.

Although more experiments are needed to nail down why the genetic architecture of temperature adaptation is highly dependent on the founder population and not on the selection regime, our results have important implications for all studies attempting to characterize the response of natural populations to climate change. The analysis of a single population cannot be sufficient to understand the genetic basis of adaptive traits. Thus, multiple diverged populations need to be studied to reach conclusions that can be generalized beyond a limited number of focal populations.

\section{Material and methods}

Unless stated otherwise, analysis was conducted using R v3.6.1 [97].

\section{Experimental populations and selection regime}

The set-up of the evolution experiment is described in detail elsewhere [46]. In brief, female flies were sampled from a natural Drosophila simulans population in Northern Portugal in the summer of 2008 and used to establish 250 isofemale lines. These lines were kept for 10 generations in the laboratory before starting the experiment. Mated females of all lines were used to create the starting populations. Ten replicates were created by combining an equal number of flies from each line. Five of the replicates were 
then kept in a $12 \mathrm{~h}: 12 \mathrm{~h}$ day and night cycle with temperatures of $20^{\circ} \mathrm{C}$ during the day and $10^{\circ} \mathrm{C}$ during the night (cold regime). The population size was kept constant at 1250 per replicate in non-overlapping generations.

The other five replicates were used to start the evolution experiment described by Mallard F, Nolte V, Tobler R, Kapun M, and Schlötterer C [46], which was identical to the cold regime except for the temperature which fluctuated between $28^{\circ} \mathrm{C}$ during the day and $18^{\circ} \mathrm{C}$ at night (hot regime).

\section{Evolve and resequence}

For the founder population, we used the sequences described by Mallard F, Nolte V, Tobler R, Kapun M, and Schlötterer C [46], but added new sequence data from two replicates of generation F3 from the cold regime to increase coverage. To avoid biases related to different sequencing approaches, all reads were pooled and then randomly split into five subsets with a coverage of $100 \times$ each. These subsets were used as founder population replicates throughout the analysis of the cold-evolved and the (re-)analysis of the hot-evolved populations. While sequence data for the F59 in the hot regime were available from Mallard F, Nolte V, Tobler R, Kapun M, and Schlötterer C [46], we added new Pool-Seq data for the intermediate time points F15 and F37 in the hot regime to allow for the time series analysis and haplotype reconstruction in this study. All sequences used for the founder population (including the F3 from the cold regime) and all sequences of the hot regime were derived from females only.

Sequencing data from the time points F11, F21, F31, F41, and F51 in the cold regime were newly generated from pooled females and males. Paired-end libraries were generated with different protocols and sequenced on different Illumina platforms (see Supplementary Table S1).

\section{Data processing}

Sequencing reads were trimmed using Readtools TrimFastq version 0.2 [98]. with the parameters --minReadLength 50 --disable5pTrim --mottQualityThreshold 20. The $D$. simulans genome sequence created by Palmieri N, Nolte V, Chen J and Schlötterer C [99] was used as reference for read mapping. To avoid false positive outlier SNPs, which might arise when libraries with different read lengths and insert sizes are combined in one analysis [100], three different mappers were used to map reads of the two time points used for outlier testing (cold regime: F0 and F51; hot regime: F0 and F59). Reads were mapped using Bowtie2 version 2.2.6 [101] with parameters --end-to-end --X 1500, bwa mem version 0.7.13 [102] with default parameters and novoalign version 3.03.2 [103] with parameters -i 350,100 -F STDFQ -o SAM -r RANDOM.

The intermediate time points (cold regime: F11, F21, F31, F41; hot regime F15, F37) used for the detection of selected haplotype blocks were mapped with novoalign only, as this mapper is known to estimate allele frequencies most accurately [100].

Mapped reads were filtered for mapping quality $\geq 20$ and proper pairs using SAMtools view version 1.3.1 [104]. Duplicates were removed using picard MarkDuplicates version 2.1.1 [105]. Barcoded files were split using Readtools AssignReadGroupByBarcode version 0.2.2 [98] with parameters --maximumMismatches 1. The BAM files were then used to create mpileup files with SAMtools mpileup version 1.3.1 [104], and finally, PoPoolation2 mpileup2sync.jar version 1.201 [106] was used to create sync files 
from mpileup files. All subsequent analysis was conducted on the basis of these sync files.

\section{SNP calling and masking}

Single nucleotide polymorphisms (SNPs) were called from the founder population by creating sync files from BAM files as described above but filtering for polymorphic sites that had a mapping quality of at least 30 and a minimal count of at least 5 and were detected by the three mapping algorithms in all founder replicates. Filtering resulted in 3.8 million SNPs that were used for further analysis. Indels were detected using the PoPoolation2 identify-indel-regions.pl script, and transposable elements were detected with repeatmasker v 1.332 [107]. Custom databases made by combining RepeatMasker database Dfam_Consensus-20181026, RepBase-20181026, and transposon_sequence_set. embl.txt from flybase.org (FB 2018_06), and search engine NCBI/RMBLAST v 2.2.27+ were used for repeats $>500 \mathrm{bp}$. All sync files were masked for these repetitive regions and for known Y chromosome translocations [108] using the PoPoolation2 filter-syncby-gtf.pl script.

\section{Correcting for different insert sizes}

To correct for false positive outlier SNPs created by libraries with different insert sizes [100], mapping results from the three different mappers for the founder (F0) and most evolved (cold regime: F51; hot regime F59) population were used. $X^{2}$ tests were conducted to compare the results of the different mappers per replicate and time point. After correcting for multiple testing using the Benjamini-Hochberg procedure [109], only SNPs that showed a consistent response across comparisons (p.adjust $\geq 0.05$ ) were kept for further analysis.

\section{Candidate SNPs}

Candidate outlier SNPs were detected in the filtered sync files created from novoalign mapping results after correcting for false positive outliers as described above. Allele frequency changes between the founder and the most evolved population were analyzed using $\mathrm{CMH}$ and $\chi^{2}$ tests which are adapted for genetic drift and pool sequencing noise as implemented in the $\mathrm{R}$ package ACER version 1.0 [49]. SNPs within the top $1 \%$ of coverage were excluded from the analysis to avoid copy number variants. Intermediate generations were included in the correction approach. Effective population size per replicate was calculated using the $\mathrm{R}$ package poolSeq version 0.3 .5 [110] with the function estimateWndNe (window size $10 \mathrm{~kb}$, method P.planI, pool size and census size 1250) and used for $\mathrm{CMH}$ and $\mathrm{x}^{2}$ tests. The $\mathrm{CMH}$ test was performed using all population replicates per time point whereas $\chi^{2}$ tests were performed for each replicate separately. All results were corrected for multiple testing (Benjamini-Hochberg). Finally, candidate SNPs detected by either test (p.adjust $<0.05$ ) were combined to include consistent responses across replicates (CMH test) and replicate-specific responses $\left(\chi^{2}\right.$ test).

\section{Selected haplotype blocks}

Selected haplotype blocks were reconstructed from candidate SNP allele frequency data of all time points and replicates using the R package haplovalidate with MNCS of 0.01 [6]. Haplotype blocks were reconstructed for all time points. As haplotype blocks might 
contain more than one selected allele, early time points (cold regime: F11, F21, and F31; hot regime: F15 and F37) were used for fine-mapping of selected haplotype blocks [56]. Here, the analysis detected the characteristic signal of reconstructed haplotype blocks with multiple selection targets, which is the presence of a single haplotype block in the most evolved generation but several reconstructed haplotype blocks when analyzing the early generations separately. Haplotype blocks from the early generations showing this pattern were included in the final analysis.

Selection coefficients for the detected haplotype blocks per replicate were computed using the allele frequency trajectories of the top 10\% outlier SNPs based on CMH and $\chi^{2}$ test result and using the poolSeq v0.3.5 function estimateSH (method LLS) [110]. Only selection coefficients with $p$-value $<0.05$ were used to calculate the median selection coefficient for each selected allele. Relationship of selection coefficient, starting allele frequency and block size were tested using a linear model with log10 transformation of selection coefficients. To test the robustness of our definition for selected alleles, we repeated the estimation of selection coefficients using (a) the top 20\% SNPs or (b) all SNPs that had an allele frequency change $>0.1$ (Additional file 1: Figure S2). Candidate genes per block were detected from the gene annotation of the reference genome [99] including also SNPs $200 \mathrm{bp}$ up- and downstream of the focal gene.

\section{Comparison to other experimentally evolved $D$. simulans populations}

We used data from the same $D$. simulans population evolving under a hot temperature regime [46] to contrast adaptation to different temperatures. For this population, we included two additional time points, so that four time points in total were available: F0, F15, F37, F59. The data set was filtered, candidate SNPs were detected and haplotypes were reconstructed in the same way as described above including the combined analysis of all (F0-F59) and early (F0-F37) time points. To estimate how many shared marker SNPs were expected by chance, we randomly sampled the number of shared SNPs from the haplotype blocks, calculated the fraction per haplotype block $(N=10,000)$, and finally applied a $95 \%$ cut-off.

In addition, a different published hot-evolved D. simulans population from Florida [45] was used for the comparative analysis. The data set was filtered and candidate SNPs were detected in the same way as described above. As intermediate time points were available for this data set (every 10th generation from F0 to F60), haplovalidate [6] with MNCS of 0.01 was used to detect selected haplotype blocks including the combined analysis of all (F0-F60) and early (F0-F30) time points as described for the Portugal population. Selection coefficients were computed as described above.

We fitted a linear model with $\log 10$ transformed selection coefficients as response and main effects of population as fixed categorical effect with three levels (Florida hot, Portugal hot, Portugal cold) and a linear and quadratic covariate for starting allele frequency, to account for their non-linear relationship with the response, as explanatory variables. Residuals from this model were normally distributed and displayed variance homogeneity. The model with linear and quadratic covariate for starting allele frequency fit significantly better than a model with only a linear term. Contrasts between populations were compared based on estimated marginal means ( $\mathrm{R}$ package emmeans).

We compared the similarity of replicates calculating the Jaccard indices for the Portugal and the Florida population using the R package philentropy [111]. We created 
binary data based on the replicate-specific selection response, i.e. whether or not a significant selection coefficient could be estimated by the poolSeq package (see above) for the corresponding replicate and allele $(p$-value $<0.05)$. Following Barghi $\mathrm{N}$, Tobler $\mathrm{R}$, Nolte V, Jaksic AM, Mallard F, Otte KA, Dolezal M, Taus T, Kofler R, and Schlötterer $\mathrm{C}$ [45], we created binary data by applying a cut-off of 0.1 to the median allele frequency change of selected alleles per replicate. Jaccard indices between populations were compared using the two-sample Wilcoxon rank-sum test. To analyze the effect of the different number of replicates (10 in the Florida and five in the Portugal population), we applied the same haplotype reconstruction pipeline as described above to all Florida subsets. However, haplotype reconstruction was not possible for 95 of 100 subsets, as no correlation signal could be detected. We interpret this as the consequence of the high redundancy resulting in different selected haplotype blocks in the replicates, which reduces the power to detect them. Hence, we repeated the analysis of selection coefficients and Jaccard indices on a downsampled set of Florida replicates. For this analysis, we took 100 random samples of five replicates from the Florida population data set and repeated the analysis for each of them as described above.

\section{Nucleotide diversity in the ancestral populations}

Nucleotide diversity $(\pi)$ of each autosome in the ancestral populations was calculated from the allele frequency data using the formula of Tajima F [112]. For maintaining a comparable number of low-frequency alleles we subsampled the Florida data set to five replicates. As different sets of five Florida replicates resulted in very consistent $\pi$ estimates (data not shown) we only used one set for the direct comparison to Portugal.

\section{Linkage disequilibrium in the ancestral populations}

To quantify linkage disequilibrium, we used 189 haplotype sequences of the Florida founder population [113] and 34 haplotype sequences of the Portugal founder population which are described in Langmüller AM, Nolte V, Galagedara R, Poupardin R, Dolezal M, Lečić S, and S. S [65]. For maintaining a comparable number of low-frequency alleles, we subsampled the Florida data set to 34 haplotypes. We calculated the mean $\mathrm{r}^{2}$ for loci (minor allele count $=3$, minimum SNP quality $=50$ ) within $10,000 \mathrm{~kb}$ distance. As different sets of 34 Florida haplotypes resulted in very consistent mean estimates (see Additional file 1: Figure S9) we only used one set for the direct comparison to Portugal.

\section{Linkage disequilibrium simulations}

We illustrated the effect of linkage equilibrium or strong linkage disequilibrium (LD) on polygenic adaptation after a shift in trait optimum with forward simulations based on Wright-Fisher populations using MimicrEE2 v208 [114]. Using the qff mode we simulated stabilizing selection on a quantitative trait based on a Gaussian fitness function and diallelic loci. We used parameters that matched the Portugal D. simulans E\&R experiment with five replicates, each starting with the same 1000 homozygous individuals which evolved for 50 generations and simulated adaptation from standing genetic variation. For computational simplicity, we simulated one genomic region of $1 \mathrm{Mb}$ containing 10 contributing loci. Each selection target started with an allele frequency of 0.05 and contributed with an additive effect size of 0.05 to the phenotype, while the other 
allele had an effect size of -0.05 . Heritability was set to $h=0.5$. We performed 100 independent simulations. Overall, the genotypic value was estimated as the sum of the effect sizes of all contributing loci while the phenotypic value was calculated by adding a Gaussian random number based on heritability $\left(h^{2}\right)$ to the genotypic value. Fitness was finally estimated based on the phenotypic value and the corresponding fitness function. We used the following Gaussian fitness function as previously described (e.g. [6]): $\mathrm{N}$ $(0.5 ; 1.2)$ scaled to a maximum of 4.5 and a minimum of 0.5 to avoid individuals with a fitness of 0 . The average genotypic value of the ancestral population was 0.45 , the average phenotypic value was 0.449 , and the average initial fitness was estimated as 3.41 . We simulated a constant recombination rate based on the average recombination rate of $1.72 \mathrm{cM} / \mathrm{Mb}$ estimated for D. simulans (Dsim_recombination_map_LOESS_100kb_1. txt, [113]).

We generated two different sets of founder populations, one with strong LD and one with linkage equilibrium. Both sets of founder populations contained loci with 200 chromosomes carrying an allele contributing to the quantitative trait towards the new trait optimum and 800 chromosomes with alleles of opposite effect. For high LD, we generated a defined haplotype structure based on four different sets of selected haplotypes. The number of contributing loci was randomly distributed between the four sets of selected haplotypes. Hence, 50 haplotypes shared the same contributing loci, but due to stochastic sampling the number of contributing loci differed among the four sets of 50 haplotypes. To generate starting populations in linkage equilibrium, we randomly distributed the contributing loci across 200 haplotypes until each of the loci had a final frequency of 0.05 . Average $r^{2}$ in these linkage equilibrium simulations ranged from 0.03 to 0.04 (see Fig. $4 \mathrm{~A}$ ).

After 50 generations, we generated "Pool-seq data" with 50x coverage and added sequencing noise by binomial sampling based on the allele frequencies. We recorded the phenotypic and mean frequency change across loci and replicates as well as the coefficient of variation in the mean allele frequency change across the five replicates as an indicator for the degree of parallel response.

\section{Supplementary Information}

The online version contains supplementary material available at https://doi.org/10.1186/s13059-021-02425-9.

Additional file 1: Figure S1. Female fecundity as proxy for fitness in the ancestral population and for each evolved replicate in the Florida and Portugal populations. Figure S2. The inverse relationship between starting allele frequency and selection coefficient is robust to the definition of the selected allele. Figure S3. Histogram of the number of genes per reconstructed haplotype block in the cold-evolved Portugal population. Figure S4. Selected haplotype blocks from replicated D. simulans populations from Portugal evolving in a novel hot environment. Figure S5. Allele frequency changes between the founder and the evolved populations. Figure S6. Selected haplotype blocks from replicated D. simulans populations from Florida evolving in a novel hot environment. Figure S7. The adaptive architecture is population-specific but does not depend on the temperature regime. Figure S8. Replicate populations derived from Portugal founders show a more similar selection response than those with Florida ancestry. Figure S9. Linkage disequilibrium in the ancestral Florida population.

Additional file 2: Table S1. Details of DNA extraction, Illumina library preparation and sequencing for all samples used in this study.

Additional file 3. Review history.

\section{Acknowledgements}

We thank Neda Barghi for sharing unpublished fecundity data and for providing information concerning the hotevolved D. simulans data set. Anna Langmüller, Thomas Taus, and Claire Burny shared code to remove SNPs with allele frequency estimates that are sensitive to the insert sizes of NGS sequencing libraries. The authors also thank the members of the Institute of Population Genetics for discussion and support on the project. Special thanks to Marlies Dolezal 
for in depth statistical advice. Illumina sequencing for a subset of the data was performed at the VBCF NGS Unit (http://www.viennabiocenter.org/facilities).

\section{Peer review information}

Kevin Pang was the primary editor of this article and managed its editorial process and peer review in collaboration with the rest of the editorial team.

\section{Review history}

The review history is available as Additional file 3.

\section{Authors' contributions}

Kathrin A Otte: Formal analysis, investigation, visualization, writing - original draft; Viola Nolte: Investigation, resources, project administration, methodology, writing - review and editing; Francois Mallard: Investigation, Writing - review and editing; Christian Schlötterer, Conceptualization, supervision, funding acquisition, writing - original draft. The authors read and approved the final manuscript.

\section{Authors' information}

Twitter handles: @kathrinaotte (Kathrin A. Otte); @FrancoisMallard (François Mallard).

\section{Funding}

KAO was supported by a DFG Research Fellowship (OT 532/1-1, OT 532/2-1 ). FM was supported by a Marie Sklodowska-Curie Individual Fellowship (H2020-MSCA-IF-661149). CS was supported by the European Research Council grant "ArchAdapt" and the Austrian Science Funds (FWF, P27630, P29133).

\section{Availability of data and materials}

Raw sequences were deposited at the European Nucleotide Archive (ENA) under the accession number PRJEB39259 [115]. Already published sequence data from Mallard et al. 2018 [46] can be found at ENA under the accession number PRJEB27022, data from Howie et al. [113] at PRJEB29483, and data from Langmüller et al. [65] at PRJEB39894. For detailed information on the already published sequence data see Additional file 2: Table S1. Population sync files, all results, and scripts were deposited on Dryad Digital Repository https://doi.org/10.5061/dryad.np5hqbzsp [116].

\section{Declarations}

Ethics approval and consent to participate

Not applicable.

\section{Competing interests}

The authors declare that they have no competing interests.

\section{Author details}

${ }^{1}$ Institut für Populationsgenetik, Vetmeduni Vienna, Vienna, Austria. ${ }^{2}$ Present address: Institute for Zoology, University of Cologne, Cologne, Germany. ${ }^{3}$ Present address: Institut de Biologie de l'École Normale Supérieure, CNRS UMR 8197, Inserm U1024, PSL Research University, F-75005 Paris, France.

Received: 26 September 2020 Accepted: 29 June 2021

Published online: 16 July 2021

\section{References}

1. Barghi N, Hermisson J, Schlötterer C. Polygenic adaptation: a unifying framework to understand positive selection. Nat Rev Genet. 2020;21:769-81. https://doi.org/10.1038/s41576-020-0250-z.

2. Flint J, Mott R. Finding the molecular basis of quantitative traits: successes and pitfalls. Nat Rev Genet. 2001;2(6):437-45. https://doi.org/10.1038/35076585.

3. McCarthy MI, Abecasis GR, Cardon LR, Goldstein DB, Little J, loannidis JP, et al. Genome-wide association studies for complex traits: consensus, uncertainty and challenges. Nat Rev Genet. 2008;9(5):356-69. https://doi.org/10.1038/nrg2344.

4. Gazal S, Finucane HK, Furlotte NA, Loh PR, Palamara PF, Liu X, et al. Linkage disequilibrium-dependent architecture of human complex traits shows action of negative selection. Nat Genet. 2017;49(10):1421-7. https:// doi.org/10.1038/ng.3954.

5. Zeng J, de Vlaming R, Wu Y, Robinson MR, Lloyd-Jones $L R$, Yengo $L$, et al. Signatures of negative selection in the genetic architecture of human complex traits. Nat Genet. 2018;50(5):746-53. https://doi.org/10.1038/s41588-018-0101-4.

6. Otte KA, Schlötterer C. Detecting selected haplotype blocks in evolve and resequence experiments. Mol Ecol Resour. 2021;21(1):93-109. https://doi.org/10.1111/1755-0998.13244.

7. Storz JF. Using genome scans of DNA polymorphism to infer adaptive population divergence. Mol Ecol. 2005;14(3):67188. https://doi.org/10.1111/j.1365-294X.2005.02437.x.

8. Vitti JJ, Grossman SR, Sabeti PC. Detecting natural selection in genomic data. Annu Rev Genet. 2013;47(1):97-120. https://doi.org/10.1146/annurev-genet-111212-133526.

9. Harris AM, DeGiorgio M. Identifying and classifying shared selective sweeps from multilocus data. Genetics. 2020;215(1): 143-71. https://doi.org/10.1534/genetics.120.303137.

10. Lee KM, Coop G. Distinguishing Among Modes of Convergent Adaptation Using Population Genomic Data. Genetics 2017;207(4):1591-619. https://doi.org/10.1534/genetics.117.300417.

11. Turner TL, Bourne EC, Von Wettberg EJ, Hu TT, Nuzhdin SV. Population resequencing reveals local adaptation of Arabidopsis lyrata to serpentine soils. Nat Genet. 2010;42(3):260-3. https://doi.org/10.1038/ng. 515. 
12. Johnson KE, Voight BF. Patterns of shared signatures of recent positive selection across human populations. Nat Ecol Evol. 2018;2(4):713-20. https://doi.org/10.1038/s41559-018-0478-6.

13. Jones FC, Grabherr MG, Chan YF, Russell P, Mauceli E, Johnson J, et al. The genomic basis of adaptive evolution in threespine sticklebacks. Nature. 2012;484(7392):55-61. https://doi.org/10.1038/nature10944.

14. Reid NM, Proestou DA, Clark BW, Warren WC, Colbourne JK, Shaw JR, et al. The genomic landscape of rapid repeated evolutionary adaptation to toxic pollution in wild fish. Science. 2016;354(6317):1305-8. https://doi.org/10.1126/science.aa h4993.

15. Roesti M, Gavrilets S, Hendry AP, Salzburger W, Berner D. The genomic signature of parallel adaptation from shared genetic variation. Mol Ecol. 2014;23(16):3944-56. https://doi.org/10.1111/mec.12720.

16. Barton NH, Keightley PD. Understanding quantitative genetic variation. Nat Rev Genet. 2002;3(1):11-21. https://doi.org/1 $0.1038 /$ nrg700

17. Sella G, Barton NH. Thinking about the evolution of complex traits in the era of genome-wide association studies. Annu Rev Genomics Hum Genet. 2019;20(1):461-93. https://doi.org/10.1146/annurev-genom-083115-022316.

18. Barton NH, Etheridge AM, Veber A. The infinitesimal model: definition, derivation, and implications. Theor Popul Biol. 2017:118:50-73. https://doi.org/10.1016/j.tpb.2017.06.001.

19. Bulmer MG. Effect of Selection on Genetic Variability. Am Nat. 1971;105:201.

20. Fisher RA. The correlation between relatives on the supposition of Mendelian Inheritance. Trans Roy Soc Edinburgh. 1918;52:339-433.

21. Field Y, Boyle EA, Telis N, Gao Z, Gaulton KJ, Golan D, et al. Detection of human adaptation during the past 2000 years. Science. 2016;354(6313):760-4. https://doi.org/10.1126/science.aag0776.

22. Jain K, Stephan W. Modes of rapid polygenic adaptation. Molecular Biology and Evolution. 2017;34(12):3169-75. https:// doi.org/10.1093/molbev/msx240.

23. Pritchard JK, Pickrell JK, Coop G. The genetics of human adaptation: hard sweeps, soft sweeps, and polygenic adaptation. Curr Biol. 2010;20(4):R208-15. https://doi.org/10.1016/j.cub.2009.11.055.

24. de Vladar HP, Barton N. Stability and response of polygenic traits to stabilizing selection and mutation. Genetics. 2014; 197(2):749-67. https://doi.org/10.1534/genetics.113.159111.

25. Jain K, Stephan W. Rapid adaptation of a polygenic trait after a sudden environmental shift. Genetics. 2017;206(1):389406. https://doi.org/10.1534/genetics.116.196972.

26. Höllinger I, Pennings PS, Hermisson J. Polygenic adaptation: from sweeps to subtle frequency shifts. PLoS Genet. 2019;15 (3):e1008035. https://doi.org/10.1371/journal.pgen.1008035

27. Conte GL, Arnegard ME, Best J, Chan YF, Jones FC, Kingsley DM, et al. Extent of QTL reuse during repeated phenotypic divergence of sympatric threespine stickleback. Genetics. 2015;201(3):1189-200. https://doi.org/10.1534/ genetics.115.182550.

28. Adeyemo A, Gerry N, Chen G, Herbert A, Doumatey A, Huang H, et al. A genome-wide association study of hypertension and blood pressure in African Americans. PLoS Genet. 2009;5(7):e1000564. https://doi.org/10.1371/journal.pgen.1000564.

29. Al Olama AA, Kote-Jarai Z, Berndt SI, Conti DV, Schumacher F, Han Y, et al. A meta-analysis of 87,040 individuals identifies 23 new susceptibility loci for prostate cancer. Nat Genet. 2014;46(10):1103-9. https://doi.org/10.1038/ng.3094.

30. Hodonsky CJ, Baldassari AR, Bien SA, Raffield LM, Highland HM, Sitlani CM, et al. Ancestry-specific associations identified in genome-wide combined-phenotype study of red blood cell traits emphasize benefits of diversity in genomics. BMC Genomics. 2020;21(1):228. https://doi.org/10.1186/s12864-020-6626-9.

31. Li YR, Keating BJ. Trans-ethnic genome-wide association studies: advantages and challenges of mapping in diverse populations. Genome Med. 2014;6(10):91. https://doi.org/10.1186/s13073-014-0091-5.

32. Takata R, Takahashi A, Fujita M, Momozawa Y, Saunders EJ, Yamada H, et al. 12 new susceptibility loci for prostate cancer identified by genome-wide association study in Japanese population. Nat Commun. 2019;10(1):4422. https://doi.org/10.1 038/s41467-019-12267-6.

33. Wojcik GL, Graff M, Nishimura KK, Tao R, Haessler J, Gignoux CR, et al. Genetic analyses of diverse populations improves discovery for complex traits. Nature. 2019;570(7762):514-8. https://doi.org/10.1038/s41 586-019-1310-4

34. Wu Y, Waite LL, Jackson AU, Sheu WH, Buyske S, Absher D, et al. Trans-ethnic fine-mapping of lipid loci identifies population-specific signals and allelic heterogeneity that increases the trait variance explained. PLoS Genet. 2013;9(3): e1003379. https://doi.org/10.1371/journal.pgen.1003379.

35. Horikoshi M, Day FR, Akiyama M, Hirata M, Kamatani Y, Matsuda K, et al. Elucidating the genetic architecture of reproductive ageing in the Japanese population. Nat Commun. 2018;9(1):1977. https://doi.org/10.1038/s41467-01804398-Z.

36. Zan Y, Carlborg O. A polygenic genetic architecture of flowering time in the worldwide Arabidopsis thaliana population. Mol Biol Evol. 2019;36(1):141-54. https://doi.org/10.1093/molbev/msy203.

37. Jensen JD, Kim Y, DuMont VB, Aquadro CF, Bustamante CD. Distinguishing between selective sweeps and demography using DNA polymorphism data. Genetics. 2005;170(3):1401-10. https://doi.org/10.1534/genetics.104.038224.

38. Johri $P$, Charlesworth B, Jensen JD. Toward an evolutionarily appropriate null model: jointly inferring demography and purifying selection. Genetics. 2020;215(1):173-92. https://doi.org/10.1534/genetics.119.303002.

39. Li J, Li H, Jakobsson M, Li S, Sjodin P, Lascoux M. Joint analysis of demography and selection in population genetics: where do we stand and where could we go? Mol Ecol. 2012;21(1):28-44. https://doi.org/10.1111/j.1365-2 94X.2011.05308.x.

40. Lohmueller KE. The impact of population demography and selection on the genetic architecture of complex traits. PLoS Genet. 2014;10(5):e1004379. https://doi.org/10.1371/journal.pgen.1004379.

41. Pavlidis P, Alachiotis N. A survey of methods and tools to detect recent and strong positive selection. J Biol Res (Thessalon). 2017;24(1):7. https://doi.org/10.1186/s40709-017-0064-0.

42. Stajich JE, Hahn MW. Disentangling the effects of demography and selection in human history. Mol Biol Evol. 2005;22(1): 63-73. https://doi.org/10.1093/molbev/msh252

43. Kawecki TJ, Lenski RE, Ebert D, Hollis B, Olivieri I, Whitlock MC. Experimental evolution. Trends Ecol Evol. 2012;27(10):54760. https://doi.org/10.1016/j.tree.2012.06.001. 
44. Garland T, Rose MR. Experimental Evolution: concepts, methods, and applications of selection experiments. Berkeley: University of California Press; 2009. https://doi.org/10.1525/9780520944473.

45. Barghi N, Tobler R, Nolte V, Jaksic AM, Mallard F, Otte KA, et al. Genetic redundancy fuels polygenic adaptation in Drosophila. PLoS Biol. 2019;17(2):e3000128. https://doi.org/10.1371/journal.pbio.3000128.

46. Mallard F, Nolte V, Tobler R, Kapun M, Schlötterer C. A simple genetic basis of adaptation to a novel thermal environment results in complex metabolic rewiring in Drosophila. Genome Biol. 2018;19(1):119. https://doi.org/10.1186/ s13059-018-1503-4.

47. Barghi N, Schlötterer C. Distinct patterns of selective sweep and polygenic adaptation in evolve and re-sequence studies. Genome Biol Evol. 2020;12(6):890-904. https://doi.org/10.1093/gbe/evaa073.

48. Schlötterer C, Tobler R, Kofler R, Nolte V. Sequencing pools of individuals - mining genome-wide polymorphism data without big funding. Nat Rev Genet. 2014;15(11):749-63. https://doi.org/10.1038/nrg3803.

49. Spitzer K, Pelizzola M, Futschik A. Modifying the Chi-square and the CMH test for population genetic inference: adapting to overdispersion. Ann Appl Stat. 2020;14:202-20.

50. Jha AR, Miles CM, Lippert NR, Brown CD, White KP, Kreitman M. Whole-genome resequencing of experimental populations reveals polygenic basis of egg-size variation in Drosophila melanogaster. Mol Biol Evol. 2015;32(10):2616-32. https://doi.org/10.1093/molbev/msv136.

51. Jha AR, Zhou D, Brown CD, Kreitman M, Haddad GG, White KP. Shared genetic signals of hypoxia adaptation in drosophila and in high-altitude human populations. Mol Biol Evol. 2016;33(2):501-17. https://doi.org/10.1093/molbev/ msv248.

52. Kelly JK, Hughes KA. Pervasive linked selection and intermediate-frequency alleles are implicated in an evolve-andresequencing experiment of Drosophila simulans. Genetics. 2019;211(3):943-61. https://doi.org/10.1534/genetics.118.301824.

53. Michalak P, Kang L, Schou MF, Garner HR, Loeschcke V. Genomic signatures of experimental adaptive radiation in Drosophila. Mol Ecol. 2019;28(3):600-14. https://doi.org/10.1111/mec.14917.

54. Franssen SU, Barton NH, Schlötterer C. Reconstruction of haplotype-blocks selected during experimental evolution. Mol Biol Evol. 2017;118:42-51.

55. Nuzhdin SV, Turner TL. Promises and limitations of hitchhiking mapping. Curr Opin Genet Dev. 2013;23(6):694-9. https:// doi.org/10.1016/j.gde.2013.10.002.

56. Otte KA, Schlötterer C. Detecting selected haplotype blocks in evolve and resequence experiments. Mol Ecol Resour. 2021;21:93-109. https://doi.org/10.1111/1755-0998.13244.

57. Eyre-Walker A. Evolution in health and medicine Sackler colloquium: Genetic architecture of a complex trait and its implications for fitness and genome-wide association studies. Proc Natl Acad Sci U S A. 2010;107(Suppl 1):1752-6. https://doi.org/10.1073/pnas.0906182107.

58. Mancuso N, Rohland N, Rand KA, Tandon A, Allen A, Quinque D, et al. The contribution of rare variation to prostate cancer heritability. Nat Genet. 2016;48(1):30-5. https://doi.org/10.1038/ng.3446.

59. Simons YB, Turchin MC, Pritchard JK, Sella G. The deleterious mutation load is insensitive to recent population history. Nat Genet. 2014;46(3):220-4. https://doi.org/10.1038/ng.2896.

60. Xu S. Theoretical basis of the Beavis effect. Genetics. 2003;165(4):2259-68. https://doi.org/10.1093/genetics/165.4.2259.

61. Beavis WD. QTL analyses: power, precision, and accuracy; 1998.

62. Bataille L, Delon I, Da Ponte JP, Brown NH, Jagla K. Downstream of identity genes: muscle-type-specific regulation of the fusion process. Dev Cell. 2010;19(2):317-28. https://doi.org/10.1016/j.devcel.2010.07.008.

63. David JR, Allemand R, Van Herrewege J, Cohet Y. Ecophysiology: abiotic factors. In: Ashburner M, Carson HL, Thompson Jr JN, editors. The Genetics and Biology of Drosophila. Volume 3d. London: Academic; 1983. p. 105-70.

64. Petavy G, David JR, Gibert P, Moreteau B. Viability and rate of development at different temperatures in Drosophila: a comparison of constant and alternating thermal regimes. J Therm Biol. 2001;26(1):29-39. https://doi.org/10.1016/S0306-4 565(00)00022-X.

65. Langmüller AM, Nolte V, Galagedara R, Poupardin R, Dolezal M, Schlötterer C. Fitness effects for Ace insecticide resistance mutations are determined by ambient temperature. BMC Biol. 2020;18(157).https://doi.org/10.1186/s12915-02 0-00882-5.

66. Charlesworth B, Charlesworth D. A study of linkage disequilibrium in populations of Drosophila melanogaster. Genetics. 1973;73(2):351-9. https://doi.org/10.1093/genetics/73.2.351.

67. Langley CH, Tobari YN, Kojima KI. Linkage disequilibrium in natural populations of Drosophila melanogaster. Genetics. 1974;78(3):921-36. https://doi.org/10.1093/genetics/78.3.921.

68. Franssen SU, Kofler R, Schlötterer C. Uncovering the genetic signature of quantitative trait evolution with replicated time series data. Heredity (Edinb). 2017;118(1):42-51. https://doi.org/10.1038/hdy.2016.98.

69. Tobler R, Franssen SU, Kofler R, Orozco-Terwengel P, Nolte V, Hermisson J, et al. Massive habitat-specific genomic response in D. melanogaster populations during experimental evolution in hot and cold environments. Mol Biol Evol. 2014;31(2):364-75. https://doi.org/10.1093/molbev/mst205.

70. Morgan TJ, Mackay TF. Quantitative trait loci for thermotolerance phenotypes in Drosophila melanogaster. Heredity (Edinb). 2006;96(3):232-42. https://doi.org/10.1038/sj.hdy.6800786.

71. Kellermann V, van Heerwaarden B, Sgro CM, Hoffmann AA. Fundamental evolutionary limits in ecological traits drive Drosophila species distributions. Science. 2009;325(5945):1244-6. https://doi.org/10.1126/science.1175443.

72. Thornton KR. Polygenic Adaptation to an Environmental Shift: Temporal Dynamics of Variation Under Gaussian Stabilizing Selection and Additive Effects on a Single Trait. Genetics. 2019;213(4):1513-30. https://doi.org/10.1534/ genetics.119.302662.

73. Shi H, Kichaev $\mathrm{G}$, Pasaniuc $B$. Contrasting the genetic architecture of 30 complex traits from summary association data. Am J Hum Genet. 2016;99(1):139-53. https://doi.org/10.1016/j.jhg.2016.05.013.

74. Visscher PM, Macgregor S, Benyamin B, Zhu G, Gordon S, Medland S, et al. Genome partitioning of genetic variation for height from 11,214 sibling pairs. Am J Hum Genet. 2007;81 (5):1104-10. https://doi.org/10.1086/522934.

75. Yang J, Manolio TA, Pasquale LR, Boerwinkle E, Caporaso N, Cunningham JM, et al. Genome partitioning of genetic variation for complex traits using common SNPs. Nat Genet. 2011;43(6):519-25. https://doi.org/10.1038/ng.823.

76. Yeaman S, Whitlock MC. The genetic architecture of adaptation under migration-selection balance. Evolution. 2011;65(7): 1897-911. https://doi.org/10.1111/j.1558-5646.2011.01269.x. 
77. Sachdeva H, Barton NH. Introgression of a block of genome under infinitesimal selection. Genetics. 2018;209(4):1279303. https://doi.org/10.1534/genetics.118.301018.

78. Gibert JM, Blanco J, Dolezal M, Nolte V, Peronnet F, Schlötterer C. Strong epistatic and additive effects of linked candidate SNPs for Drosophila pigmentation have implications for analysis of genome-wide association studies results. Genome Biol. 2017;18(1):126. https://doi.org/10.1186/s13059-017-1262-7.

79. King EG, Merkes CM, MCNeil CL, Hoofer SR, Sen S, Broman KW, et al. Genetic dissection of a model complex trait using the Drosophila Synthetic Population Resource. Genome Res. 2012;22(8):1558-66. https://doi.org/10.1101/gr.134031.111.

80. Shahandeh MP, Turner TL. The complex genetic architecture of male mate choice evolution between Drosophila species. Heredity (Edinb). 2020;124(6):737-50. https://doi.org/10.1038/s41437-020-0309-9.

81. Kerdaffrec E, Filiault DL, Korte A, Sasaki E, Nizhynska V, Seren U, et al. Multiple alleles at a single locus control seed dormancy in Swedish Arabidopsis. Elife. 2016;5. https://doi.org/10.7554/eLife.22502.

82. Stam LF, Laurie CC. Molecular dissection of a major gene effect on a quantitative trait: the level of alcohol dehydrogenase expression in Drosophila melanogaster. Genetics. 1996;144(4):1559-64. https://doi.org/10.1093/ genetics/144.4.1559.

83. Zan Y, Sheng Z, Lillie M, Ronnegard L, Honaker CF, Siegel PB, et al. Artificial selection response due to polygenic adaptation from a multilocus, multiallelic genetic architecture. Mol Biol Evol. 2017;34(10):2678-89. https://doi.org/10.1 093/molbev/msx194.

84. Baldwin-Brown JG, Long AD, Thornton KR. The power to detect quantitative trait Loci using resequenced, experimentally evolved populations of diploid, sexual organisms. Mol Biol Evol. 2014;31(4):1040-55. https://doi.org/10.1093/molbev/ msu048.

85. Kofler R, Schlötterer C. A guide for the design of evolve and resequencing studies. Mol Biol Evol. 2014;31(2):474-83. https://doi.org/10.1093/molbev/mst221.

86. Oomen RA, Kuparinen A, Hutchings JA. Consequences of single-locus and tightly linked genomic architectures for evolutionary responses to environmental change. J Hered. 2020;111(4):319-32. https://doi.org/10.1093/jhered/esaa020.

87. Zhang XS, Hill WG. Predictions of patterns of response to artificial selection in lines derived from natural populations. Genetics. 2005;169(1):411-25. https://doi.org/10.1534/genetics.104.032573.

88. Hoffmann AA. Physiological climatic limits in Drosophila: patterns and implications. J Exp Biol. 2010;213(6):870-80. https://doi.org/10.1242/jeb.037630.

89. Chevin LM, Lande R, Mace GM. Adaptation, plasticity, and extinction in a changing environment: towards a predictive theory. PLOS Biol. 2010;8(4):e1000357. https://doi.org/10.1371/journal.pbio.1000357.

90. Kopp M, Matuszewski S. Rapid evolution of quantitative traits: theoretical perspectives. Evol Appl. 2014;7(1):169-91. https://doi.org/10.1111/eva.12127.

91. Bridle JR, Gavaz S, Kennington WJ. Testing limits to adaptation along altitudinal gradients in rainforest Drosophila. Proc Biol Sci. 2009;276:1507-15.

92. Hoffmann AA, Anderson A, Hallas R. Opposing clines for high and low temperature resistance in Drosophila melanogaster. Ecology Letters. 2002;5(5):614-8. https://doi.org/10.1046/j.1461-0248.2002.00367.x.

93. Rako L, Blacket MJ, McKechnie SW, Hoffmann AA. Candidate genes and thermal phenotypes: identifying ecologically important genetic variation for thermotolerance in the Australian Drosophila melanogaster cline. Mol Ecol. 2007;16(14): 2948-57. https://doi.org/10.1111/j.1365-294X.2007.03332.x.

94. Bergland AO, Behrman EL, O'Brien KR, Schmidt PS, Petrov DA. Genomic evidence of rapid and stable adaptive oscillations over seasonal time scales in Drosophila. PLoS Genet. 2014;10(11):e1004775. https://doi.org/10.1371/journal.pgen.1004775.

95. Kemppainen P, Li Z, Rastas P, Löytynoja A, Fang B, Yang J, Guo B, Shikano T, Merilä J. Genetic population structure constrains local adaptation in sticklebacks. Mol Ecol. 2021;30:1946-61. https://doi.org/10.1111/mec.15808

96. Seabra SG, Fragata I, Antunes MA, Faria GS, Santos MA, Sousa VC, et al. Different genomic changes underlie adaptive evolution in populations of contrasting history. Mol Biol Evol. 2018;35(3):549-63. https://doi.org/10.1093/molbev/msx247.

97. R: A Language and Environment for Statistical Computing [http://www.r-project.org/index.html].

98. Gomez-Sanchez D, Schlötterer C. ReadTools: A universal toolkit for handling sequence data from different sequencing platforms. Mol Ecol Resour. 2017.

99. Palmieri N, Nolte V, Chen J, Schlötterer C. Genome assembly and annotation of a Drosophila simulans strain from Madagascar. Mol Ecol Resour. 2015;15(2):372-81. https://doi.org/10.1111/1755-0998.12297.

100. Kofler R, Langmüller AM, Nouhaud P, Otte KA, Schlötterer C. Suitability of Different Mapping Algorithms for GenomeWide Polymorphism Scans with Pool-Seq Data, G3. 2016;6(11):3507-15. https://doi.org/10.1534/g3.116.034488.

101. Langmead B, Salzberg SL. Fast gapped-read alignment with Bowtie 2. Nat Methods. 2012;9(4):357-9. https://doi.org/10.1 038/nmeth.1923.

102. Li H, Durbin R. Fast and accurate long-read alignment with Burrows-Wheeler transform. Bioinformatics. 2010;26(5):58995. https://doi.org/10.1093/bioinformatics/btp698.

103. Novocraft: NovoAlign. 2014.

104. Li H, Handsaker B, Wysoker A, Fennell T, Ruan J, Homer N, et al. The Sequence Alignment/Map format and SAMtools. Bioinformatics. 2009;25(16):2078-9. https://doi.org/10.1093/bioinformatics/btp352.

105. Broad Institute: Picard toolkit. 2019. http://broadinstitute.github.io/picard/.

106. Kofler R, Pandey RV, Schlötterer C. PoPoolation2: identifying differentiation between populations using sequencing of pooled DNA samples (Pool-Seq). Bioinformatics. 2011;27(24):3435-6. https://doi.org/10.1093/bioinformatics/btr589.

107. Smit AFA, Hubley R, Green P: RepeatMasker Open-4.0 (2013-2015). 2015. (http://www.repeatmasker.org/).

108. Tobler R, Nolte V, Schlotterer C. High rate of translocation-based gene birth on the Drosophila Y chromosome. Proc Natl Acad Sci U S A. 2017;114(44):11721-6. https://doi.org/10.1073/pnas.1706502114.

109. Benjamini $Y$, Hochberg Y. Controlling the false discovery rate: a practical and powerful approach to multiple testing. J R Stat Soc Ser. 1995;57:289-300

110. Taus T, Futschik A, Schlötterer C. Quantifying Selection with Pool-Seq Time Series Data. Mol Biol Evol. 2017;34(11):302334. https://doi.org/10.1093/molbev/msx225.

111. Drost HG. Philentropy: information theory and distance quantification with R. Journal of Open Source Software. 2018;3 (26):765. https://doi.org/10.21105/joss.00765. 
112. Tajima F. Statistical method for testing the neutral mutation hypothesis by DNA polymorphism. Genetics. 1989;123(3): 585-95. https://doi.org/10.1093/genetics/123.3.585.

113. Howie JM, Mazzucco R, Taus T, Nolte V, Schlotterer C. DNA motifs are not general predictors of recombination in two Drosophila sister species. Genome Biol Evol. 2019;11(4):1345-57. https://doi.org/10.1093/gbe/evz082.

114. Vlachos C, Kofler R. MimicrEE2: Genome-wide forward simulations of Evolve and Resequencing studies. PLoS Comput Biol. 2018;14(8):e1006413. https://doi.org/10.1371/journal.pcbi.1006413.

115. Otte KA, Nolte V, Schlötterer C. The genetic architecture of temperature adaptation is shaped by population ancestry and not by selection regime. Datasets. European Nucleotide Archive. 2021. https:/wwww.ebi.ac.uk/ena/browser/view/PRJEB39259.

116. Otte KA, Nolte V, Schlötterer C. The genetic architecture of temperature adaptation is shaped by population ancestry and not by selection regime. Dryad Digital Repository. 2021. https://doi.org/10.5061/dryad.np5hqbzsp.

\section{Publisher's Note}

Springer Nature remains neutral with regard to jurisdictional claims in published maps and institutional affiliations.

\section{Ready to submit your research? Choose BMC and benefit from:}

- fast, convenient online submission

- thorough peer review by experienced researchers in your field

- rapid publication on acceptance

- support for research data, including large and complex data types

- gold Open Access which fosters wider collaboration and increased citations

- maximum visibility for your research: over $100 \mathrm{M}$ website views per year

At $\mathrm{BMC}$, research is always in progress. 